\title{
Possibilities of Using Frequency Energy Functions in Problems of Dynamics of Machines for Transport and Technological Purposes
}

\author{
Andrey Eliseev ${ }^{1,2, *}$, Nikolay Kuznetsov ${ }^{1}$, Sergey Eliseev², and Roman Bolshakov² \\ ${ }^{1}$ Irkutsk National Research Technical University, 664074, Irkutsk, Russia \\ ${ }^{2}$ Irkutsk State Transport University, 664074, Irkutsk, Russia
}

\begin{abstract}
New approaches to the evaluation of the dynamic properties of mechanical vibration systems are proposed, which consider as the design schemes for technical objects operating under dynamic vibration loads. The purpose of the study is to develop ideas for the use of frequency energy functions. In this case, the frequency functions represent the ratio of the potential and kinetic energies in a specific form, based on the use of the relations of the coordinates of the system in the stationary mode. The technologies of system analysis and structural mathematical modeling are used. In this approach, the mechanical oscillatory system is correspond with the block diagram of the automatic control system, which is equivalent in dynamic terms. It is proposed to use the analytical apparatus to take into account the features of interpartial relations, to evaluate the specific modes of dynamic interaction of system elements. A method for determining the natural vibration frequencies of the system based on the use of the frequency energy function is proposed. Methodological bases of approaches to solving problems of system dynamics related to the assessment of the influence of system parameters on their frequency properties are developed. The results of computational modeling are presented.
\end{abstract}

\section{Introduction}

Many technical objects of transport and technological purposes are operated in conditions of increased dynamic loads. This requires appropriate efforts at the stages of preliminary research, calculations and selection of structural and technical calculations for the reliability of operation and safety of operation of technical means. Much attention is paid to the dynamics of machines operating under conditions of intense dynamic vibration loads [1-3]. In this connection, the directions associated with vibration protection and vibration isolation of machines, devices and equipment [4-6], as well as modes of dynamic vibration damping [7, 8], specific forms of connectivity of the movement of system elements, as well as other specific modes [9] are highlighted. Various studies are aimed at creating a

*Corresponding author: Eliseev_AV@irgups.ru 
scientific and methodological basis for solving the problem of the dynamics of machines, equipment and mechanisms operating under periodic (most often harmonic) external influences of a power or kinematic nature. In this connection, attention is justified to the features of dynamic properties that can be reflected using the frequency characteristics of systems. These characteristics give an idea of resonant phenomena and dynamic vibration damping. This is of great importance for the formation of parameters of the dynamic quality of systems, operating safety conditions, etc. The determination of the natural vibration frequencies of systems involves the construction of mathematical models based on analytical or approximate approaches, which is usually used in engineering practice [10, 11].At the same time, there is a need for more detailed estimates of the frequency properties of mechanical oscillatory systems, which in analytical approaches encounters certain difficulties. Interesting ideas in this direction were proposed by Rayleigh [12].

The proposed report reflects a scientific and methodological approach that allows us to consider the frequency properties of mechanical oscillatory systems in a fairly generalized form and evaluate the possible forms of influence of the system parameters on the natural frequencies of its free oscillations.

\section{Some general provisions}

Many technical objects, including railway and automobile transport, as well as vibrating technological machines (vibration stands, vibration transporters, etc.), can be considered in the first approximation on the basis of design schemes in the form of mechanical oscillatory systems with concentrated parameters with two degrees of freedom. It is assumed that the system has linear properties and makes small fluctuations relative to the position of static equilibrium or steady motion. Fig. 1, a shows the design scheme in the form of a mechanical oscillatory system of the chain type with two degrees of freedom.
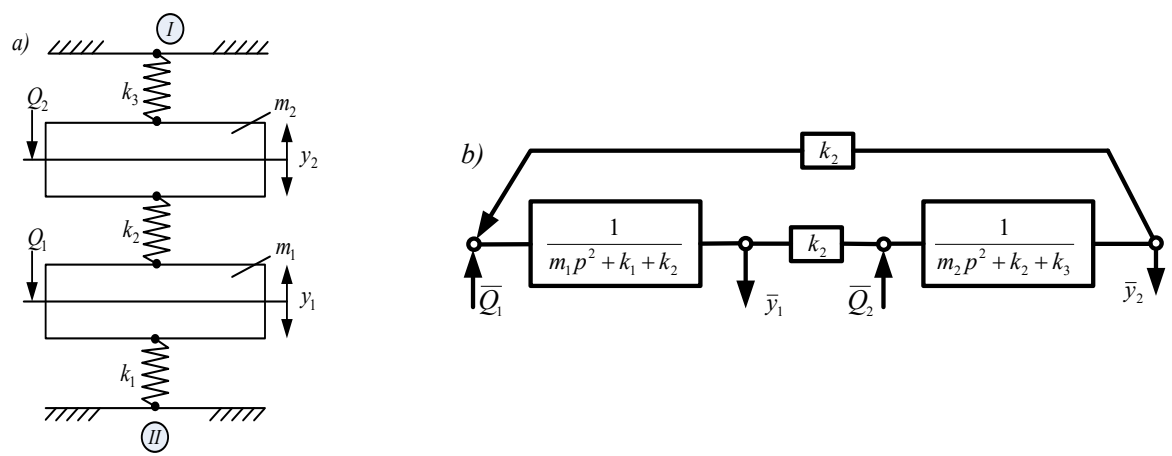

Fig. 1. Design $(a)$ and structural $(b)$ schemes of a linear mechanical oscillatory system with two degrees of freedom

In turn, Fig. 1, $b$ shows a structural mathematical model of a dynamically equivalent automatic control system.

Using the calculation scheme in Fig. 1, $a$, which defines the properties of a linear system with two degrees of freedom $\left(y_{1}, y_{2}\right)$, it is possible to obtain a mathematical model in the form of a system of two linear differential equations of the second order with constant coefficients based on the application of Lagrange equations of the second kind. To do this, it is necessary to obtain an expression for the kinetic and potential energy, which is done in the usual ways, for example, according to the method described in [3,9]. After the Laplace transformations under zero initial conditions, the original system of equations of motion in the coordinates $y_{1}, y_{2}$ can be transformed to a system of equations in operator 
form, on the basis of which a structural mathematical model can be constructed (Fig. 1, $b$ ) in the form of a block diagram of a dynamically equivalent automatic control system. As it follows from the design scheme in Fig. 1, a, the design scheme in the form of a chain mechanical system consists of two mass-inertia elements $m_{1}, m_{2}$ and three elastic links with stiffness coefficients $k_{1}-k_{3}$; the system is affected by two harmonic external forces $Q_{1}$ and $Q_{2}$; the motion is described in the coordinate system $y_{1}, y_{2}$, associated with a fixed basis.

Using the expressions for the kinetic and potential energies using the Lagrange equation of the second kind, the equations of motion can be constructed in the coordinates $y_{1}, y_{2}$. We apply the Laplace transformations under zero initial conditions. The resulting system of linear differential equations with constant coefficients can be transformed to an operator form, which ultimately allows us to construct the block diagram shown in Fig. $1, b$ ( $p=j \omega$-is a complex variable; the $\left.<^{-}\right\rangle$icon above the variable means its image according to Laplace [13]. Using the block diagram in Fig. 1, $b$ can write an expression for the transfer function of the system.

For transport objects and a number of technological vibration machines, a typical design scheme is a mechanical oscillatory system with an object whose dynamic state is estimated in the form of a solid body with a mass $M$ and a moment of inertia $J$; the solid body is supported by elastic supports with stiffness coefficients $k_{1}$ and $k_{2}$.

Fig. 2,a,b shows the corresponding calculation scheme (see Fig. 2, a) and the block diagram (see Fig. 2,b).
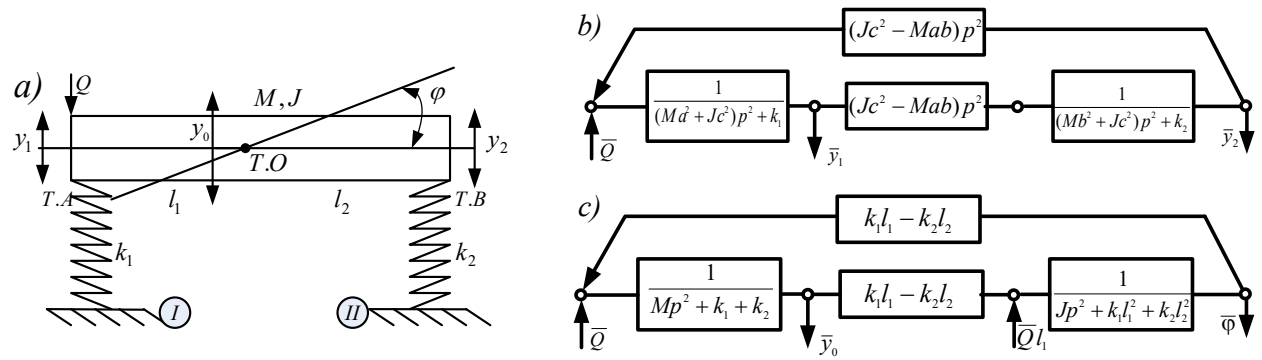

Fig. 2. Design $(a)$ and structural $(b, c)$ schemes of a system with a solid body with two degrees of freedom: $a$-design scheme with an object in the form of a solid body; $b$-structural scheme of the system in coordinates $y_{1}$ and $y_{2} ; c$-structural scheme of the system in coordinates $y_{0}$ and $\varphi$

\section{Features of mathematical modeling in problems of estimating the natural vibration frequencies of a system}

For the system in Fig. 1,a, the expressions for the kinetic and potential energies in the coordinates $y_{1}$ and $y_{2}$ have the form

$$
\begin{aligned}
& \mathrm{T}=\frac{1}{2} m_{1} \dot{y}_{1}^{2}+\frac{1}{2} m_{2} \dot{y}_{2}^{2}, \\
& \Pi=\frac{1}{2} k_{1} y_{1}^{2}+\frac{1}{2} k_{2}\left(y_{2}-y_{1}\right)^{2}+\frac{1}{2} k_{3} y_{3}^{2} .
\end{aligned}
$$

In this case, the frequency energy function is represented as the ratio of the potential to the kinetic energy, taking into account that the ratio between the coordinates $y_{1}$ and $y_{2}$ will be determined by some statement of the value $y_{2}=a_{0} y_{1}$. 
It is assumed that an external perturbation is applied to the element $m_{1}\left(Q_{1} \neq 0, Q_{2}=0\right)$; assuming also that the joint motions along $y_{1}$ and $y_{2}$ are harmonic, we can write

$$
\omega^{2}\left(a_{0}\right)=\frac{k_{1}+k_{2}+a_{0}^{2}\left(k_{2}+k_{3}\right)-2 k_{2} a_{0}}{m_{1}+a_{0}^{2} m_{2}} .
$$

Note that for $a_{0}=0$ :

$$
\omega^{2}\left(a_{0}\right)=\frac{k_{1}+k_{2}}{m_{1}}
$$

accordingly, at $a_{0} \rightarrow \infty$ :

$$
\omega^{2}\left(a_{0}\right)=\frac{k_{2}+k_{3}}{m_{2}}
$$

For specific values of $k_{1}, k_{2}, k_{3}, m_{1}$, and $m_{2}$, the extreme values of $\omega^{2}\left(a_{0}\right)$ can be determined by counting $a_{0}$ as an independent variable. In general, for a system with two degrees of freedom, the numerator and denominator (3) are second-order polynomials and have an extreme value. From (3), we can find the value $a_{0}$, which corresponds to the frequency of natural oscillations, as shown in Fig. 3.

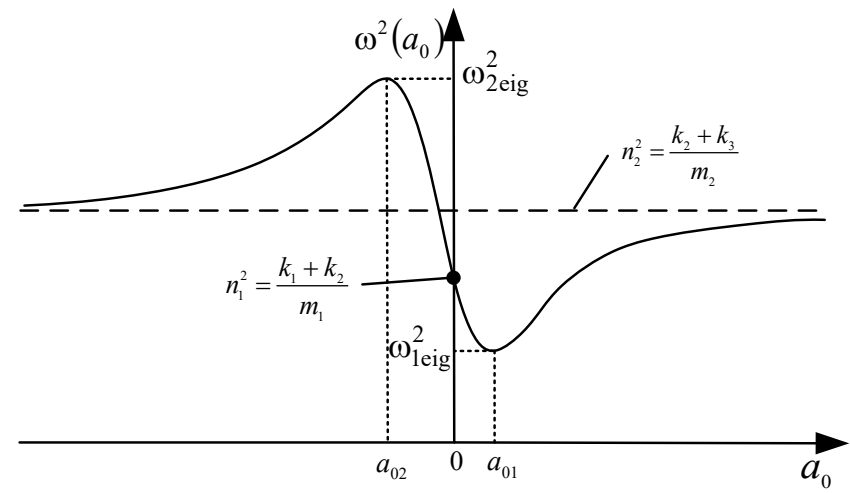

Fig. 3. Graph of the frequency energy function as a function of the amplitude distribution coefficient in the oscillation forms

The extreme value of the function $\omega^{2}\left(a_{0}\right)$ can be found in this case and analytically, from the condition

$$
\left[\omega^{2}\left(a_{0}\right)\right]^{\prime}=0
$$

where the roots have the form

$$
a_{0}=\frac{m_{2}\left(k_{1}+k_{2}\right)-m_{1}\left(k_{2}+k_{3}\right)}{2 k_{2} m_{2}} \pm \sqrt{\frac{\left[m_{1}\left(k_{2}+k_{3}\right)-m_{2}\left(k_{1}+k_{2}\right)\right]^{2}+4 m_{1} m_{2} k_{2}^{2}}{4\left(k_{2} m_{2}\right)^{2}}} .
$$

Since 


$$
a_{0}=\frac{k_{2}}{-m_{2} \omega^{2}+k_{2}+k_{3}},
$$

then, after substituting (7) in (3) or (8), we obtain expressions for the natural oscillation frequencies

$$
\omega_{1, \text { e ig }}^{2}=\frac{m_{1}\left(k_{2}+k_{3}\right)+m_{2}\left(k_{1}+k_{2}\right)}{2 m_{1} m_{2}} \pm \sqrt{\frac{\left[m_{1}\left(k_{2}+k_{3}\right)-m_{2}\left(k_{1}+k_{2}\right)\right]^{2}+4 m_{1} m_{2} k_{2}^{2}}{4\left(m_{1} m_{2}\right)^{2}}} \cdot
$$

From the analysis of the graph $\omega^{2}\left(a_{0}\right)$ in Fig. 3, it follows that the extreme values of $a_{0}$ (respectively, the points $a_{01}$ and $a_{02}$ on the abscissa axis) determine the values of the natural oscillation frequencies $\omega_{1 \text { eig }}$ and $\omega_{2 \text { eig. }}$. For $a_{0}=0$, the intersection of the curve $\omega^{2}\left(a_{0}\right)$ with the ordinate axis determines the partial frequency $n_{12}=\left(k_{1}+k_{2}\right) / m_{1}$. The branches of the curve $\omega^{2}\left(a_{0}\right)$ in the first and fourth squares of the coordinate plane have asymptotes determined by the values of the partial frequency $n_{22}=\left(k_{2}+k_{3}\right) / m_{2}$. Fig. 4 shows the results of numerical calculations for six variants of parameter values $k_{1}=k, k_{2}=2 k, k_{3}$ $=\{3 k, 5 k, 10 k\}, m_{1}=m$ and $m_{2}=\{m, 2 m\}$. The relative position of the curves $\omega^{2}\left(a_{0}\right)$ gives an idea of the influence of $k_{3}$ on the relative position of the characteristic points.

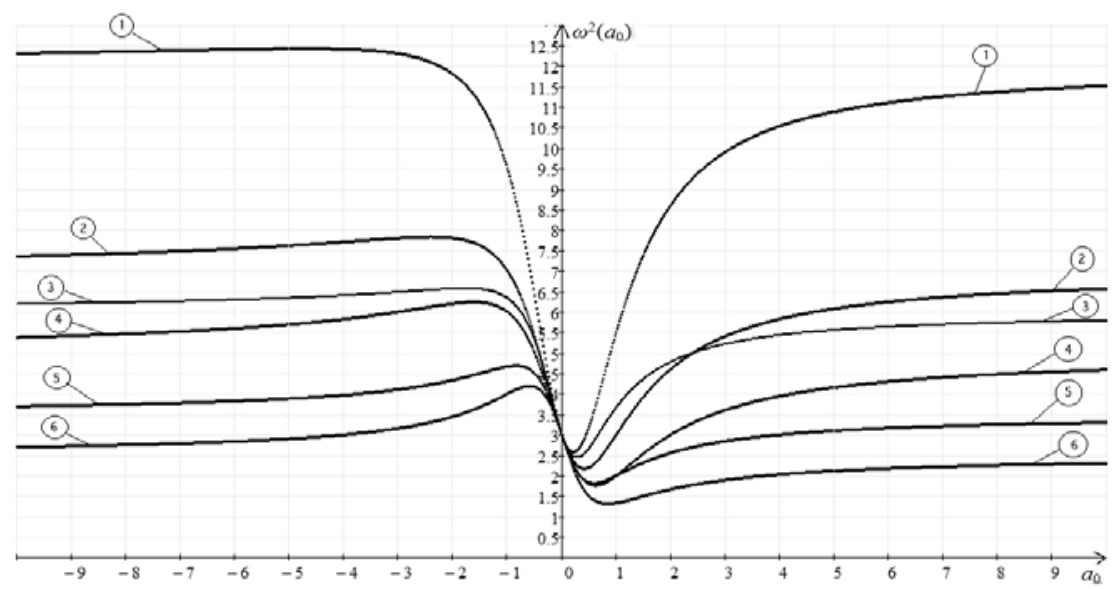

Fig. 4. Graphs of the frequency energy function as a function of the amplitude distribution coefficient in different values of masses and stiffness $\left(m_{1}=m, k_{1}=k, k_{2}=2 k\right): 1-$ at $m_{2}=m, k_{3}=10 k ; 2-$ at $m_{2}=m, k_{3}=5 k ; 3-$ at $m_{2}=m, k_{3}=3 k ; 4-$ at $m_{2}=2 m, k_{3}=10 k ; 5-$ at $m_{2}=2 m, k_{3}=5 k ; 6-$ at $m_{2}$ $=2 m, k_{3}=3 k$

\section{On the possibilities of expanding the application of the frequency energy function in mechanical oscillatory systems with a solid body performing angular oscillations on elastic supports}

The design scheme of the system is shown in Fig. 2, $a, b, c$ consists of an object with massinertia parameters $M$ (mass) and $J$ (moment of inertia), based on elastic elements with stiffness $k_{1}, k_{2}$. The motion of the system can be considered in the coordinate system $y_{1}$ and $y_{2}$, as well as in the coordinate system associated with the motion of the center of mass of a solid body $y_{0}$ and the angle of rotation $\varphi$ relative to the center of mass. 
Using the methods of structural mathematical modeling [3, 8], we construct the structural schemes of the original system (Fig. 2, a), which are shown in Fig. 2, b, c.

In the coordinate system $y_{1}$ and $y_{2}$, , the interpartial link represented by the link with the transfer function $\left(J c^{2}-M a b\right) p^{2}$ is called inertial. Under the condition $J c^{2}=M a b$-partial systems do not depend on each other, and the system splits into two independent blocks. In the coordinate system $y_{0}$ and $\varphi$, the interpartial connection is elastic. If the conditions $k_{1} l_{1}=$ $k_{2} l_{2}$ are met, then the connection between the partial systems decays. External influences in Fig. 2, b, that is, in the coordinates $y_{1}$ and $y_{2}$, are represented by the force $\bar{Q}$ applied at point $A$.

When switching to the coordinate system $y_{0}$ and $\varphi$, the condition of equality of the work of forces on possible displacements of the generalized coordinates is taken into account. In this regard, in the block diagram (Fig. 2, b) of the system in coordinates $y_{0}, \varphi$, there are two generalized forces $Q$ at the center of gravity, and the moments of forces or pairs of forces $Q l_{1}=M a b$ relative to the center of gravity.

The system is considered in the coordinates $y_{1}$ and $y_{2}$. In this case, from the block diagram in Fig. 2, b, you can determine the transfer functions of the system, which take the form:

$$
\begin{aligned}
& W_{1}(p)=\frac{\bar{y}_{1}}{\bar{Q}}=\frac{\left(M b^{2}+J c^{2}\right) p^{2}+k_{2}}{A_{1}(p)}, \\
& W_{2}(p)=\frac{\bar{y}_{2}}{\bar{Q}}=\frac{\left(J c^{2}-M a b\right) p^{2}}{A_{1}(p)},
\end{aligned}
$$

where

$$
A_{1}(p)=\left[\left(M a^{2}+J c^{2}\right) p^{2}+k_{1}\right] \cdot\left[\left(M b^{2}+J c^{2}\right) p^{2}+k_{2}\right]-\left(J c^{2}-M a b\right)^{2} p^{4}
$$

The transfer function of the interpartial link is defined by the expression

$$
W_{\bar{y}_{2}, \bar{y}_{1}}(p)=\frac{\bar{y}_{2}}{\bar{y}_{1}}=\frac{\left(J c^{2}-M a b\right) p^{2}}{\left(M b^{2}+J c^{2}\right) p^{2}+k_{2}} .
$$

The expression for the frequency energy function in this case takes the form

$$
\omega^{2}\left(a_{1}\right)=\frac{k_{1}+k_{2} a_{1}^{2}}{M a^{2}+J c^{2}+\left(M b^{2}+J c^{2}\right) a_{1}^{2}+2\left(M a b-J c^{2}\right) a_{1}} .
$$

The characteristic modes in the coordinate system are related to the following relations:

a) for $a_{1}=0, \omega^{2}\left(a_{1}\right)=\frac{k_{1}}{M a^{2}+J c^{2}}$; 
b) for a $\rightarrow \infty, \omega^{2}\left(a_{1}\right)=\frac{k_{2}}{M b^{2}+J c^{2}}$.

The partial frequencies in the coordinate system $y_{1}$ and $y_{2}$ have the form

$$
\begin{aligned}
& n_{1}^{\prime 2}=\frac{k_{1}}{M a^{2}+J c^{2}}, \\
& n_{2}^{\prime 2}=\frac{k_{2}}{M b^{2}+J c^{2}} .
\end{aligned}
$$

We introduce the transfer function between partial connections

$$
W_{\bar{\varphi}, \bar{y}_{0}}(p)=\frac{\bar{\varphi}}{\bar{y}_{0}}=\frac{l_{1} M p^{2}+2 k_{1} l_{1}+k_{2}\left(l_{1}-l_{2}\right)}{J p^{2}+2 k_{1} l_{1}^{2}-k_{2} l_{2}\left(l_{1}-l_{2}\right)} .
$$

Compared to the previous case, where, defined by expression (13), is a dimensionless quantity, and in the coordinate system $y_{0}$ and $\varphi$, the ratio (17) has the dimension rad $/ \mathrm{m}$. In this coordinate system, the lever connection is transformed into a screw connection and can be shown by a virtual screw lever.

\section{Conclusion}

The proposed report presents the results of research on the development of scientific and methodological foundations of system analysis and structural mathematical modeling in the problems of dynamics of transport and vibration technological machines under conditions of intense dynamic loading. It is shown that the design schemes of technical objects, displayed by the design schemes in the form of mechanical oscillatory systems, allow us to detail the ideas about the dynamic properties associated with the assessment of the dynamic states of objects determined by the spectrum of natural vibration frequencies in a wide range. The growing attention to the issues of ensuring the reliability of operation and safety of operation of machinery and equipment determines the attention to the frequency properties of systems and taking into account the features of their formation and manifestation. A method for estimating the dynamic properties of mechanical oscillatory systems based on the introduction of the concept of the frequency energy function is proposed. This function allows you to determine the frequencies of natural oscillations and their dependences on the system parameters, depending on the coupling coefficient of the oscillation amplitudes along the coordinates of the system. The properties of the coupling coefficients of the oscillations can be useful when introducing interpartial coupling in the transfer function. The construction of frequency characteristics in the form of a dependence of the value of the frequency energy function on the coefficient of coupling of oscillations allows us to evaluate the causes of possible changes in the dynamic properties of the system under harmonic external disturbances. The extreme values of the frequency energy function determine the value of the natural oscillation frequencies and their relationship to the partial frequencies of the systems. The limit values of the frequency function reflect the possibilities in the formation of dynamic states of objects under vibration influences. The 
scientific interest is caused by the manifestation of the relationship between the frequency of natural oscillations and the value of the interpartial coupling coefficient for free oscillations. This allows us to assume the presence of the physical property of preserving the shape of free vibrations as a certain stable property, reflecting the existence of processes of "retaining" the shape of free vibrations as a certain parameter that characterizes the interaction of elements of vibrational structures.

The reporting study was carried out with the financial support of the Ministry of science and higher education of the Russian Federation in the framework of project No. 0667-2020-0037 «Development of scientific foundations of effective processes obtaining valuable elements, substances and materials from waste and man-made and natural multicomponent mixtures, including methods of design and technological support.

\section{References}

1. C.W. De Silva, Vibration. Fundamentals and Practice (DC: CRC Press, Boca Raton, London, New York, Washington, 2000)

2. I.A. Karnovsky, E. Lebed, Theory of vibration protection (Springer, Switzerland, 2016)

3. S.V. Eliseev, A.V. Eliseev, Theory of Oscillations. Structural Mathematical Modeling in Problems of Dynamics of Technical Objects (Springer International Publishing, Cham, 2020)

4. V.S. Ilyinsky, Protection of apparatuses from dynamic impacts (Energia, Moscow, 1970)

5. V.A. Ivovich, Vibration protection in mechanical engineering (Mechanical Engineering, Moscow, 1990)

6. M.Z. Kolovsky, Automatic control of vibration protection systems (Nauka, Moscow, 1976)

7. B.G. Korenev, L.M. Reznikov, Dynamic vibration dampers. Theory and technical applications (Nauka, Moscow, 1988)

8. S.V. Eliseev, A.P. Khomenko, Dynamic vibration damping: feedback concept and structural methods of mathematical modeling (Nauka, Novosibirsk, 2014)

9. S.V. Eliseev, Applied system analysis and structural mathematical modeling (dynamics of transport and technological machines: connectivity of movements, vibration interactions, lever connections) (Irkutsk State Transport University, Irkutsk, 2018)

10. V.N. Chelomey, Vibrations in technology. Vibrations of linear systems (Mechanical Engineering, Moscow, 1978)

11. C.M. Harris, C.E. Crede, Shock and Vibration Handbook (McGraw - Hill Book Co, New York, 2002)

12. J.W. Rayleigh, Theory of sound. Vol. 1(Gostekhteoretizdat, Moscow, Leningrad, 1940)

13. A. I. Lurie, Operational calculus and application in technical applications (Nauka, Moscow, 1959) 